\title{
The Effects of Mismatch between SPECT and CT Images on Quantitative Activity Estimation - A Simulation Study
}

\section{Yingqing Lyu}

University of Macau

\section{Yue Chen}

Southwest Medical University

Greta Mok ( $\sim$ gretamok@um.edu.mo)

University of Macau https://orcid.org/0000-0002-2962-2087

\section{Original research}

Keywords: misregistration, targeted radionuclide therapy, SPECT/CT, attenuation correction, segmentation

Posted Date: June 22nd, 2021

DOl: https://doi.org/10.21203/rs.3.rs-619011/v1

License: (c) (i) This work is licensed under a Creative Commons Attribution 4.0 International License.

Read Full License 
1 The Effects of Mismatch between SPECT and CT Images on

2 Quantitative Activity Estimation - A Simulation Study

$4 \quad{ }^{1}$ Biomedical Imaging Laboratory (BIG), Department of Electrical and

5 Computer Engineering, Faculty of Science and Technology, University of

6 Macau, Taipa, Macau SAR, China

$7 \quad{ }^{2}$ Department of Nuclear Medicine, The Affiliated Hospital of Southwest

8 Medical University; Nuclear Medicine and Molecular Imaging Key

9 Laboratory of Sichuan Province, Luzhou, Sichuan, China

$10{ }^{3}$ Center for Cognitive and Brain Sciences, Institute of Collaborative

11 Innovation, University of Macau, Taipa, Macau SAR, China

12

13 * Correspondence: gretamok@um.edu.mo. 
20 Background: Quantitative activity estimation is essential in targeted radionuclide therapy 21 dosimetry. Misregistration between SPECT and CT images at the same imaging time point due to 22 patient movement degrades accuracy. This work aims to study the mismatch effects between CT 23 and SPECT data on attenuation correction (AC), volume-of-interest (VOI) delineation and 24 registration for activity estimation.

25 Methods: Nine 4D XCAT phantoms were generated at 1, 24, and 144 hrs post In-111 Zevalin 26 injection, varying in activity distributions, body and organ sizes. Realistic noisy SPECT projections 27 were generated by an analytical projection and reconstructed with quantitative OS-EM method. CT 28 images were shifted from -5 to 5 voxels as well as according to clinical reference corresponding to 29 SPECT images at each time point. For AC effect, mismatched CT images were used for AC in 30 SPECT reconstruction while VOIs were mapped out from matched CTs. For VOI effect, target 31 organs were mapped out using mismatched CTs with matched CTs for AC. For registration effect, 32 non-rigid registrations were performed on sequential mismatched CTs to align corresponding 33 SPECT images, with no AC and VOI mismatch. Bi-exponential curve fitting was performed to 34 obtain time-integrated activity (TIA). Organ activity errors (\%OAE) and TIA errors (\%TIAE) were 35 calculated.

36 Results: According to clinical reference, \%OAE was larger for organs near ribs for AC effect, e.g., $37-2.58 \% \pm 0.81 \%$ for liver. For VOI effect, \%OAE was larger for small and low uptake organs, e.g., $38-11.94 \% \pm 10.34 \%$ for spleen. $\% \mathrm{OAE}$ was proportional to mismatch magnitude, e.g., $4.77 \% \pm 1.41 \%$, $3912.01 \% \pm 3.97 \%$ and $42.81 \% \pm 6.38 \%$ for 1 -, 2 -, and 5 -voxel mismatch for lungs. For registration 40 effect, \%TIAE were larger when mismatch existed in more numbers of SPECT/CT images, while 41 no substantial difference was observed when using mismatched CT at different time points for 42 registration reference. \%TIAE was highest for VOI, followed by registration and AC, e.g., $43 \quad 37.61 \% \pm 5.08 \%, 14.25 \% \pm 7.07 \%$ and $1.13 \% \pm 0.90 \%$ respectively for kidneys. 
44 Conclusions: The mismatch between CT and SPECT images poses a significant impact on 45 accuracy of quantitative activity estimation in dosimetry, attributed particularly from VOI 46 delineation errors. It is recommended to perform registration between emission and transmission 47 images at the same time point to ensure dosimetric accuracy.

48

49 Keywords: misregistration, targeted radionuclide therapy, SPECT/CT, attenuation correction, 50 segmentation. 


\section{1. Introduction}

52 Targeted radionuclide therapy (TRT) is an effective therapy for various types of cancers [1],

53 using radionuclides labeled molecules to kill cancer cells with ionizing radiation by destroying their

54 DNA in the cell nucleus and by "bystander" effects [2], resulting in tumor shrinkage. Compared to

55 conventional chemotherapy, TRT aims to specifically deliver a lethal dose targeting cancerous cells

56 with minimal collateral toxicity to the surrounding normal organs or tissues, which requires

57 accurate drug biodistribution information prior and post treatment. Such information can be

58 obtained by planar imaging or emission computed tomography, i.e., SPECT and PET [3].

59 Sequential quantitative activity images at different time points or sometimes single time point for

60 Y-90 radioembolization allow the estimation of time-integrated activity (TIA) for critical organs

61 and tumors by fitting of time-activity curves (TACs) and obtain the area under the curves for further

62 dose conversion.

63 Integrated $\mathrm{CT}$ in SPECT/CT can be used for attenuation correction (AC) in SPECT

64 reconstruction to improve quantitative accuracy, and provide anatomical reference for the activity

65 uptakes in general. For TRT dosimetry, CT data can be further used in segmentations of tumors

66 and critical organs, or registrations to reduce the misalignments among serial scans $[4,5]$. However,

67 the accuracy of registration between SPECT and CT data is limited by certain voluntary and

68 involuntary motion since CT scans take couple seconds while SPECT imaging needs at least several

69 minutes. Voluntary variables are mainly due to patient movement between SPECT and CT scans

70 as the patient position may change during the two acquisitions. Involuntary movements are mostly

71 physiological activities, such as the beating of the heart, the peristalsis of the bowel [6] and the

72 respiratory movements of the lungs and adjacent organs [7]. Respirations cause organ movement

73 or deformation particularly in the upper abdominal [8] and lower thoracic regions [9, 10]. The

74 SPECT and CT mismatch may manifest as patients may practice breath holding during CT scans,

75 while SPECT images are acquired during free breathing, leading to data at different respiratory 
76 positions being acquired. Such movements alter the appearance of organ shape, size, and location

77 between the SPECT and CT imaging sessions, being responsible for the minor mismatches.

78 Voluntary and involuntary mismatch errors not only cause artifacts in the SPECT data due to the

79 use of misaligned CT for AC [11, 12], but also misleading anatomical localization for later

80 segmentation required for dosimetric calculations. He et al. have studied the impact of

81 SPECT/planar and CT misregistration at the same imaging time point and mis-definition of

82 volume-of-interest (VOI) from manual segmentation on activity estimation [13]. In their study,

83 VOI misregistration errors were generally larger than mis-definition errors, producing a

84 considerable source of errors on activity estimations. For planar images, the quantitation error could

85 be up to $8 \%$ for the kidneys with only 1 voxel VOI mismatch in z-direction between planar and CT

86 images. Although SPECT images were less sensitive to the VOI misregistration errors than the

87 planar images, the error was about $-5 \%$ for the spleen if SPECT and CT images had 1 voxel VOI

88 mismatch in y-direction. One limitation of their study is that only mismatch within 1 voxel, i.e.,

$894.42 \mathrm{~mm}$, between SPECT and CT is evaluated, whereas the mean misregistration for integrated

90 SPECT/CT at the same time point could reach $10.2 \pm 4.3 \mathrm{~mm}$ for 3 directions, with a range of $0-25.1$

$91 \mathrm{~mm}$ [14].

92 Besides, for serial imaging sessions, CT images are usually used for registration and the 93 resultant motion field will be used to align the corresponding SPECT images to reduce the 94 misalignments at different time points [15]. Thus, a mismatch of SPECT and CT at the same time 95 point would lead to TIA estimation errors when using CT images for registration reference. All the 96 aforementioned errors will propagate to final TIA estimation, and are expected to accumulate for 97 more number of imaging time points.

98 This study aims to systematically evaluate the impact of mismatch between CT and SPECT 99 images in the following aspects using simulations: (i) AC; (ii) VOI definition; and (iii) registration 100 among sequential SPECT/CT images. 


\section{Materials and Methods}

\section{2.A. Phantom Population}

104 A population of nine 4D digital extended cardiac torso (XCAT) phantoms was used [16]. The 105 XCAT phantoms with highly detailed body anatomies and physiological functions were generated 106 using non-uniform rational B-spline (NURBS) and subdivision surfaces based on segmentation of 107 patient datasets. It is an important imaging tool that allows modeling with user-defined parameters, 108 i.e., anatomical variations, cardiac and respiratory motion, generating realistic multimodal imaging 109 data close to the clinical studies. The phantoms used in this study varied in three anatomical 110 variations with three respective In-111 Zevalin activity distributions (Figure 1), modeling an axial 111 respiratory motion of $20 \mathrm{~mm}$ and $5 \mathrm{~s}$ period as well as normal cardiac motion. The activity 112 distribution in the background remainder, kidneys, spleen, liver, heart, bone marrow and blood 113 vessel was uniform except for the lungs since there was no airways activity. The time-varying 114 activity and effective half-life of each organ were based on a set of clinical patient data [17] to 115 simulate whole body SPECT scans covering from the thorax to the abdomen at 3 time points, i.e., 1161,24 , and $144 \mathrm{hrs}$ post-injection. 


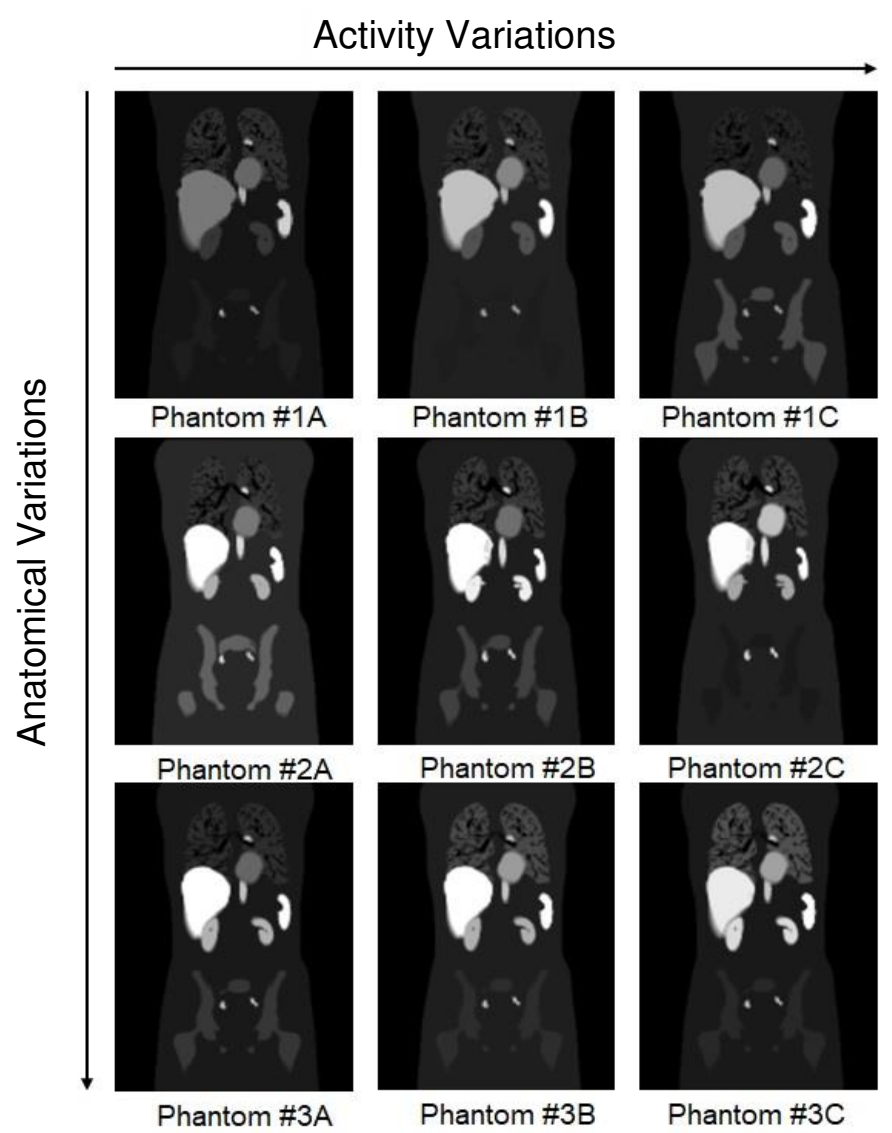

118 Figure 1: Sample activity maps of the 9 XCAT phantoms.

\section{B. Simulation and Quantitative Reconstruction}

An analytical projector of a medium energy general purpose (MEGP) collimator modeling

122 attenuation, scatter, and geometric collimator-detector-response (GCDR) was used [18] for the

123 simulations based on a GE Discovery VH Hawkeye SPECT/CT system with crystal thickness of

$1242.54 \mathrm{~cm}$. Considering two photopeaks of In-111, i.e., $171 \mathrm{keV}$ and $245 \mathrm{keV}$ with abundances of

$12590.2 \%$ and $94 \%$ respectively, the attenuation maps with abundance weighted average energy of 210

$126 \mathrm{keV}$ for $\mathrm{AC}$ in reconstruction were generated [19]. The scatter was modeled by the effective source

127 scatter estimation (ESSE) method [20] and the GCDR was performed using an analytic formulation

128 proposed by Metz et al [21]. 
The noise-free projections were generated in 128 transaxial and 170 axial bins with $4.42 \mathrm{~mm}$

130 voxel size and 128 views over $360^{\circ}$ acquisition, using phantoms with a voxel size of $2.21 \mathrm{~mm}$. A

131 system calibration factor of $1.43 \times 10^{-4}$ counts $S^{-1} B q^{-1}$ was used to scale the projections to a

132 clinical count level of $30 \mathrm{~s} /$ view acquisition time, which were then added with Poisson noise to

133 obtain realistic noisy projections. The data were reconstructed using the OS-EM algorithm (8

134 iterations and 16 subsets, i.e., 128 updates) with attenuation, ESSE and GCDR compensation.

136 2.C. Experimental Design

\section{2.C.1. The Mismatched AC Effect}

138 The SPECT/CT mismatch effect on AC was evaluated on 1 time point for organ activity and

139 3-time point for TIA estimation, respectively.

140 2.C.1.a. Organ activity estimation

141 SPECT and its corresponding CT images at $24 \mathrm{hr}$ time point for 9 phantoms were used for 142 analysis. The CT image was first shifted from 0 to 5 voxels $(0-22.1 \mathrm{~mm})$ in anterior-to-posterior $143(\mathrm{y}-)$, superior-to-inferior $(\mathrm{z}-)$ and lateral $(\mathrm{x}-)$ directions respectively as compared to the 144 corresponding SPECT image. The increments were 0.1 voxel for 0 to 1 voxel, 0.2 voxel for 1 to 2 145 voxels and 1 voxel for 2 to 5 voxels respectively. We also evaluated the random mismatch between 146 CT and SPECT images where CT images were shifted randomly within the mean Euclidian distance 147 of $10.2 \pm 4.3 \mathrm{~mm}(2.31 \pm 0.97$ voxels $)$ and a maximum range of $25.1 \mathrm{~mm}$ (5.67 voxels) as compared 148 to the corresponding SPECT images according to a clinical reference [14]. The noisy projections 149 were reconstructed using the mismatched CT maps for AC (Figure 2). VOIs of the target organs, 150 i.e., kidneys, spleen, liver and lungs were segmented from the matched CT images semi151 automatically using an open-source software application ITK-SNAP [22] to map out the 152 corresponding organs from the SPECT images to measure the organ activities. 

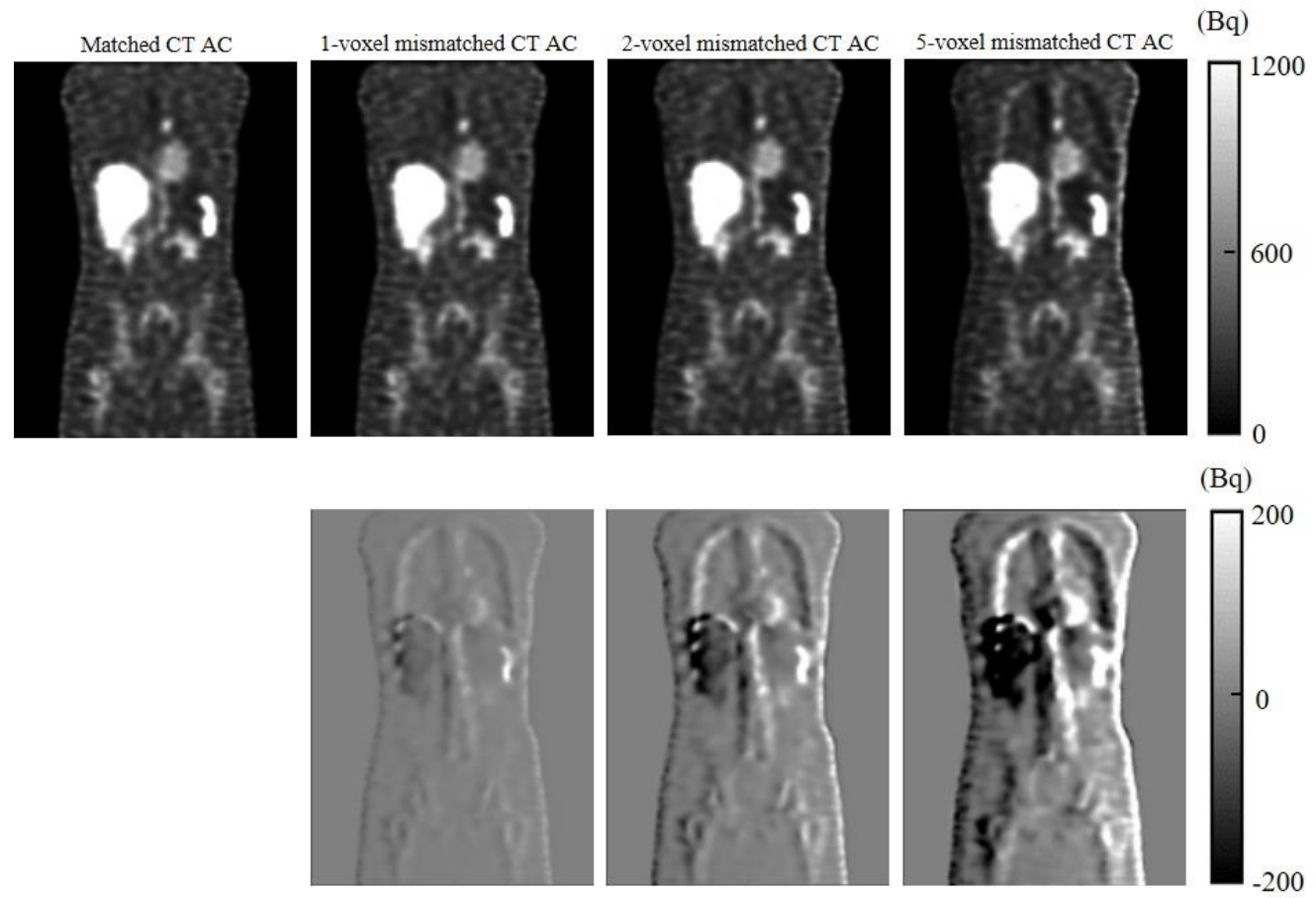

154 Figure 2: Top: Sample SPECT images using different CT maps for AC; Bottom: Difference images 155 as compared to the SPECT images using matched CT for AC.

SPECT and CT images at 1, 24, and $144 \mathrm{hr}$ time points for 9 phantoms were used for 159 evaluations, while misalignments were not modelled among images at different time points. CT images at 3 time points were shifted randomly within the mean and maximal range of 2.31 voxels and 5.67 voxels respectively as compared to the corresponding SPECT images, which were then used for AC for the corresponding SPECT reconstructions (Figure 3). Voxel-based bi-exponential curve fitting using the nonlinear least squares method under MATLAB 9.6 (The MathWorks Inc.,

164 Natick, USA) was performed on the sequential quantitative SPECT images over 3 time points to 165 obtain the TIA images, i.e., area under the curves by integration. VOIs segmented from the matched 166 CT images were used to map out the target organs from the TIA images. 


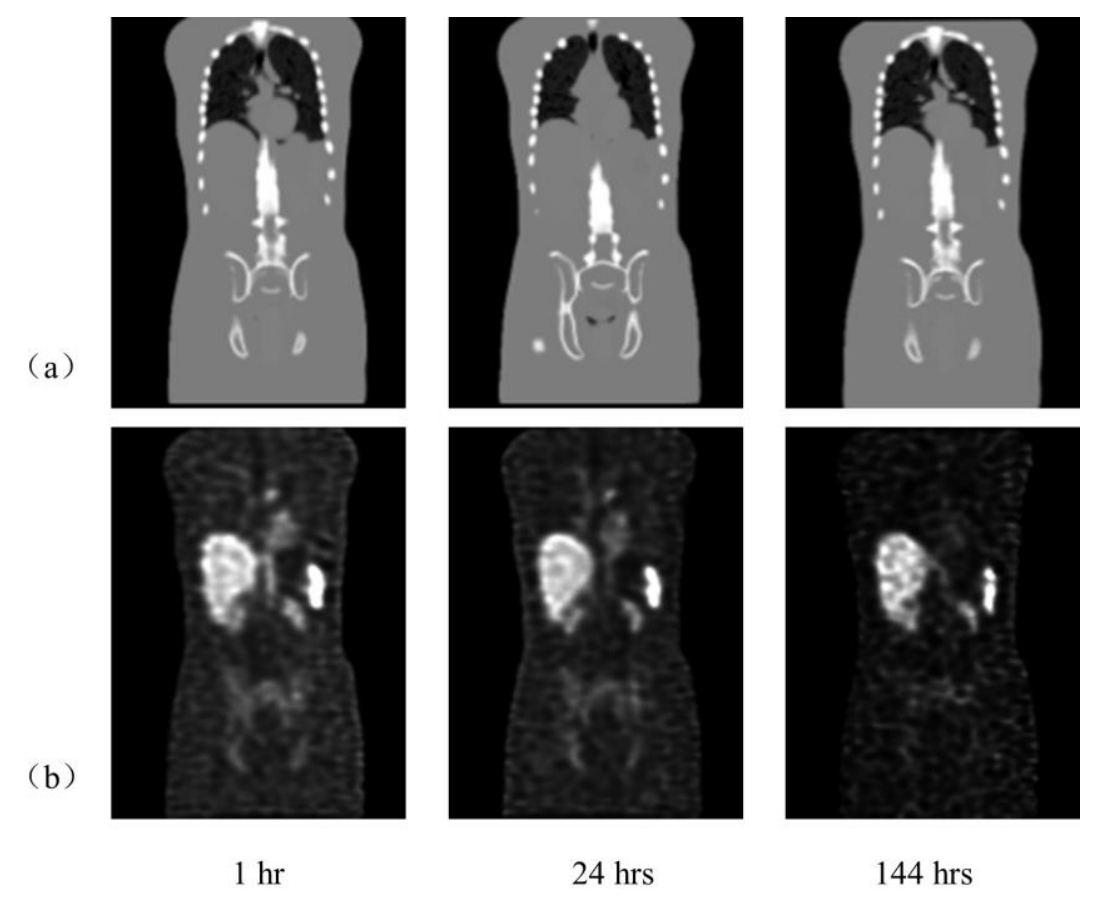

168 Figure 3: The mismatched AC effect for TIA estimation: (a) mismatched CT images and (b) the 169 corresponding SPECT images at 3 different time points.

\section{2.C.2. The Mismatched VOI Delineation Effect}

172 Similar to the previous section, the mismatch effect on VOI delineation was investigated on 173 organ activity and TIA estimation errors.

174 2.C.2.a. Organ activity estimation

175 The simulated noisy SPECT projections at 24-hr time point were reconstructed using the 176 matched CT maps for AC. Then the target organs on SPECT images were mapped out using 177 mismatched VOIs obtained from the shifted CT, generated as described in Section 2.C.1.a, to obtain 178 the organ activities.

179 2.C.2.b. TIA estimation 
The simulated noisy projections at 3 time points were reconstructed with corresponding matched CT maps for AC. Misalignments were not modeled among different time points.

182 Considering the VOIs may apply on the sequential SPECT images or the TIA image directly, VOI 183 mismatch was modeled in 1 and 3 time points respectively. For the 3-time-point VOI mismatch, 184 target organs were mapped out from the SPECT image at each time point using the mismatched 185 VOIs from shifted CT images as described in Section 2.C.1.b. Curve fitting and integration were 186 then performed as described in the previous section on the segmented target organs to obtain their 187 TIA. For the 1-time-point VOI mismatch, curve fitting and integration were first performed on the 188 sequential SPECT images to obtain the TIA images. The target organs were then mapped out using mismatched VOI at 24-hr time point from the TIA images.

\section{C.3. The Mismatched Registration Effect}

To model the misalignment among sequential scans, the local organ deformation was performed for kidneys, spleen, liver, and stomach at 1 and $144 \mathrm{hrs}$ time point, translated and rotated randomly within \pm 5 pixels $(11.05 \mathrm{~mm})$ or degrees, while using the $24 \mathrm{hrs}$ time point as the reference.

195 The volume change was held within 5\% for each organ except the stomach. The boundaries of the 196 lungs were defined by the deformation of surrounding organs, i.e., liver and heart. Besides the local 197 organ deformation, to simulate the whole body movement between scans, a rigid transformation 198 within \pm 5 pixels or degrees of translation or rotation was also modeled [23, 24].

Sequential CT images of the 9 phantoms were shifted randomly as compared to their corresponding SPECT images for 1-, 2- and 3-time points respectively as described in 2.C.1.b. CT images were then non-rigidly registered to the "reference image", i.e., 24-hr CT images, using the affine plus B-spline framework under the open-source program "Elastix" [25]. The resultant motion 
vectors were then applied to align the corresponding SPECT images with matched CT AC. The TIA images were derived by curve fitting and integration of the SPECT images over 3 time points, 206 using the same method as described in the previous sections. VOIs segmented from the matched 207 CT images at the 24-hr time point were used to map out the target organs from the TIA images.

Sequential CT images of the 9 phantoms were shifted randomly as compared to their corresponding SPECT images on 1, 24 or $144 \mathrm{hr}$-time point separately. The shifted CT images at 3

211 different time points were used as "reference image" respectively for registrations. The resultant

212 motion fields were used to align the corresponding SPECT images with matched CT AC. The effect

213 of using different time point CT images as registration reference was also evaluated for sequential

214 SPECT/CT images with no SPECT and CT mismatch for comparison. Curve fitting and integration 215 were performed on the "registered" images to obtain the TIA images, and TIAs for target organs 216 were then mapped out using matched VOIs at the reference time point.

\section{$218 \quad$ 2.D. Data Analysis}

219 For the errors in organ activity estimation introduced by mismatched SPECT and CT images

220 at 1 time point caused by AC and VOI delineation, the target organ activities $\left(\boldsymbol{A}_{\boldsymbol{w}} / \mathbf{m i s m a t c h}\right)$ were 221 compared to the reference $\left(\boldsymbol{A}_{\text {ref }}\right)$, i.e., organ activities obtained with no misalignment between 222 SPECT and CT images to calculate the organ activity errors (\%OAE):

$$
\% O A E=\frac{A_{w / m i s m a t c h}-A_{r e f}}{A_{\text {ref }}} \times 100 \%
$$


227 absolute TIA error (\%TIAE) for the target organs $\left(\boldsymbol{T I} \boldsymbol{A}_{\boldsymbol{w}} /\right.$ mismatch $)$ was defined as the following 228 equation, using sequential SPECT and CT images with no SPECT/CT mismatch and no 229 misalignment between different time points for registration as reference $\left(\boldsymbol{T I} \boldsymbol{A}_{\text {ref }}\right)$ :

$$
\% T I A E=\frac{\left|T I A_{w / m i s m a t c h}-T I A_{r e f}\right|}{\text { TIA }_{\text {ref }}} \times 100 \%
$$

The \% TIAE obtained from Section 2.C.1.b, 2.C.2.b and 3-time-point mismatch for registration 232 in 2.C.3.a were compared to demonstrate the error magnitudes from AC, VOI delineation and 233 registration for TIA measurement caused by SPECT and CT mismatch. Statistical analysis was 234 performed using the paired t-test with Bonferonni correction by SPSS Version 24 (IBM Corp., 235 Armonk, NY, USA) for \%TIAE. A p value < 0.05 was defined as significantly different.

\section{3. Results}

\section{A. The Mismatched AC Effect}

Figure 4 shows the \%OAE for target organs by the mismatched AC effect for Phantom \#2A. The \%OAE for the mismatch between SPECT and CT images along $\mathrm{x}$ - and z-directions were larger than y-direction for all target organs especially for spleen, liver and lungs, e.g., \%OAE for spleen at 5 voxels were $-17.04 \%, 3.81 \%$ and $-19.55 \%$ for $\mathrm{x}-, \mathrm{y}-$, $\mathrm{z}$-direction respectively. The mismatch of attenuation maps had larger errors for organs near ribs, e.g., spleen and lungs. The \%OAE results of 9 phantoms were consistent (Table 1). The errors are generally $<3 \%$ for mismatch according to the clinical reference. 

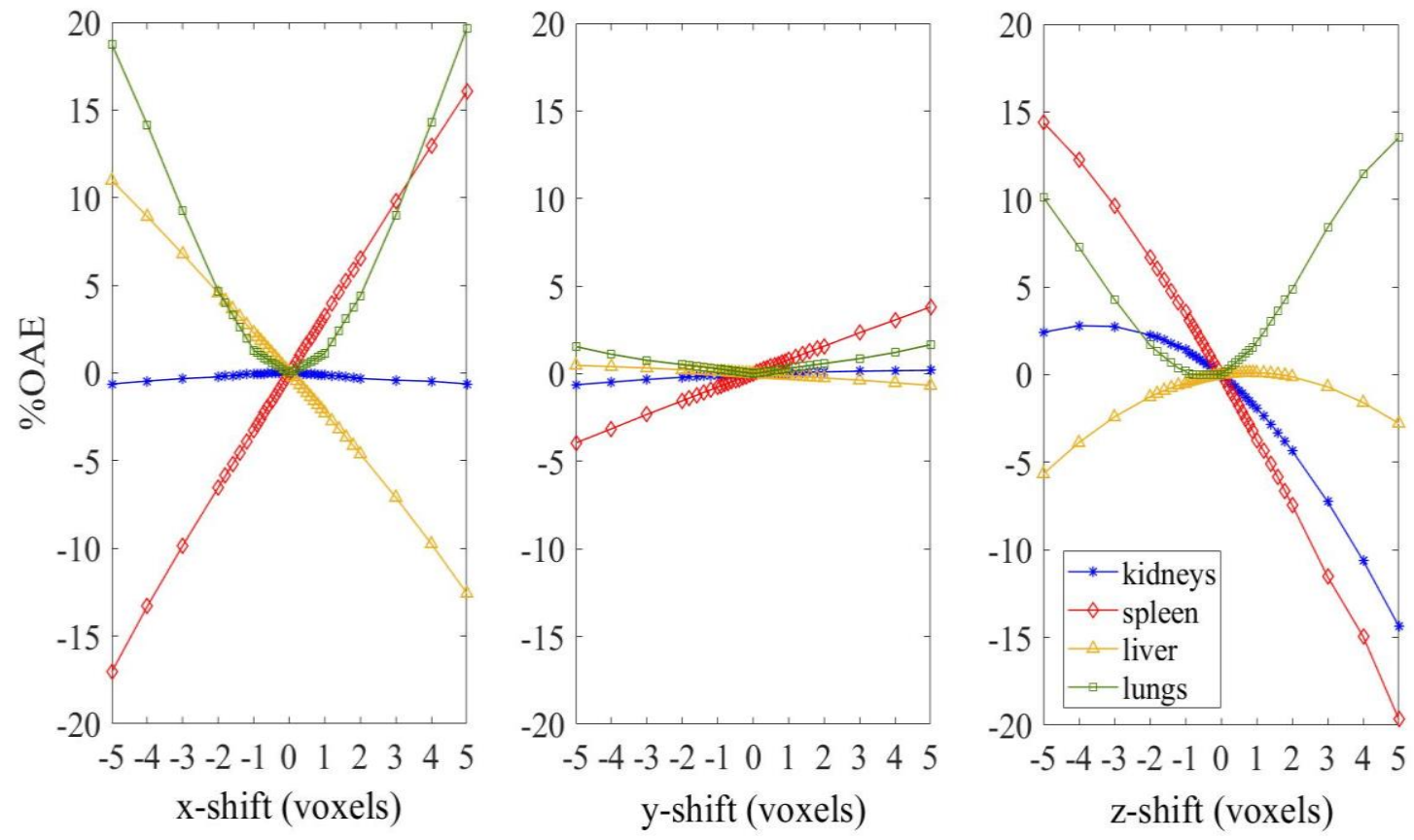

246

247 Figure 4: \%OAE introduced by shifting CT images from -5 to 5 voxels for the AC effects. Left: $\mathrm{x}-$

248 direction; middle: $y$-direction; right: $\mathrm{z}$-direction.

250 Table 1: Mean \%OAE and standard deviation of 9 phantoms for the mismatched AC effect.

\begin{tabular}{|l|l|c|c|c|c|}
\hline \multicolumn{2}{|c|}{} & kidneys & spleen & liver & lungs \\
\hline \multirow{2}{*}{$\mathrm{x}$-shift } & -1 voxel & $-0.11 \% \pm 0.06 \%$ & $-4.01 \% \pm 0.35 \%$ & $2.73 \% \pm 0.20 \%$ & $1.17 \% \pm 0.18 \%$ \\
\cline { 2 - 6 } & -2 voxels & $-0.32 \% \pm 0.12 \%$ & $-8.00 \% \pm 0.71 \%$ & $5.46 \% \pm 0.41 \%$ & $4.25 \% \pm 0.56 \%$ \\
\cline { 2 - 6 } & -5 voxels & $-1.61 \% \pm 1.25 \%$ & $-20.20 \% \pm 1.60 \%$ & $13.43 \% \pm 1.10 \%$ & $17.30 \% \pm 2.40 \%$ \\
\hline y-shift & -1 voxel & $-0.20 \% \pm 0.07 \%$ & $-0.37 \% \pm 0.19 \%$ & $0.20 \% \pm 0.04 \%$ & $0.19 \% \pm 0.10 \%$ \\
\cline { 2 - 6 } & -2 voxels & $-0.40 \% \pm 0.13 \%$ & $-0.83 \% \pm 0.33 \%$ & $0.31 \% \pm 0.08 \%$ & $1.06 \% \pm 0.49 \%$ \\
\cline { 2 - 6 } & -5 voxels & $-0.96 \% \pm 0.25 \%$ & $-2.28 \% \pm 0.76 \%$ & $0.55 \% \pm 0.19 \%$ & $4.38 \% \pm 2.29 \%$ \\
\hline$z-s h i f t$ & -1 voxel & $1.54 \% \pm 0.35 \%$ & $3.43 \% \pm 0.17 \%$ & $-0.20 \% \pm 0.15 \%$ & $0.41 \% \pm 0.09 \%$ \\
\cline { 2 - 6 } & -2 voxels & $2.30 \% \pm 0.58 \%$ & $5.62 \% \pm 0.54 \%$ & $-0.62 \% \pm 0.30 \%$ & $1.67 \% \pm 0.14 \%$ \\
\cline { 2 - 6 } & -5 voxels & $3.03 \% \pm 1.21 \%$ & $11.84 \% \pm 1.38 \%$ & $-3.90 \% \pm 0.84 \%$ & $9.86 \% \pm 1.35 \%$ \\
\hline \multicolumn{2}{|l}{$\begin{array}{l}\text { Mismatch according } \\
\text { to clinical reference }\end{array}$} & $\mathbf{- 0 . 7 2 \%} \pm \mathbf{0 . 5 0} \%$ & $\mathbf{2 . 1 3 \%} \pm \mathbf{1 . 3 4 \%}$ & $\mathbf{- 2 . 5 8 \% \pm \mathbf { 0 . 8 1 } \%}$ & $\mathbf{1 . 5 4 \%} \pm \mathbf{0 . 2 8 \%}$ \\
\hline
\end{tabular}


Figure 5 shows the $\% \mathrm{OAE}$ for the mismatched VOI delineation effects for Phantom \#2A.

255 The \%OAE for VOI effect was larger on small organs, i.e., kidneys and spleen, and organs with

256 lower activity uptake, e.g., lungs. The results show that the mismatch errors were proportional to

257 the magnitude of the CT mismatches. The \%OAE results of 9 phantoms were consistent (Table 2).

258 The errors could reach $>10 \%$ for spleen for mismatch according to the clinical reference.
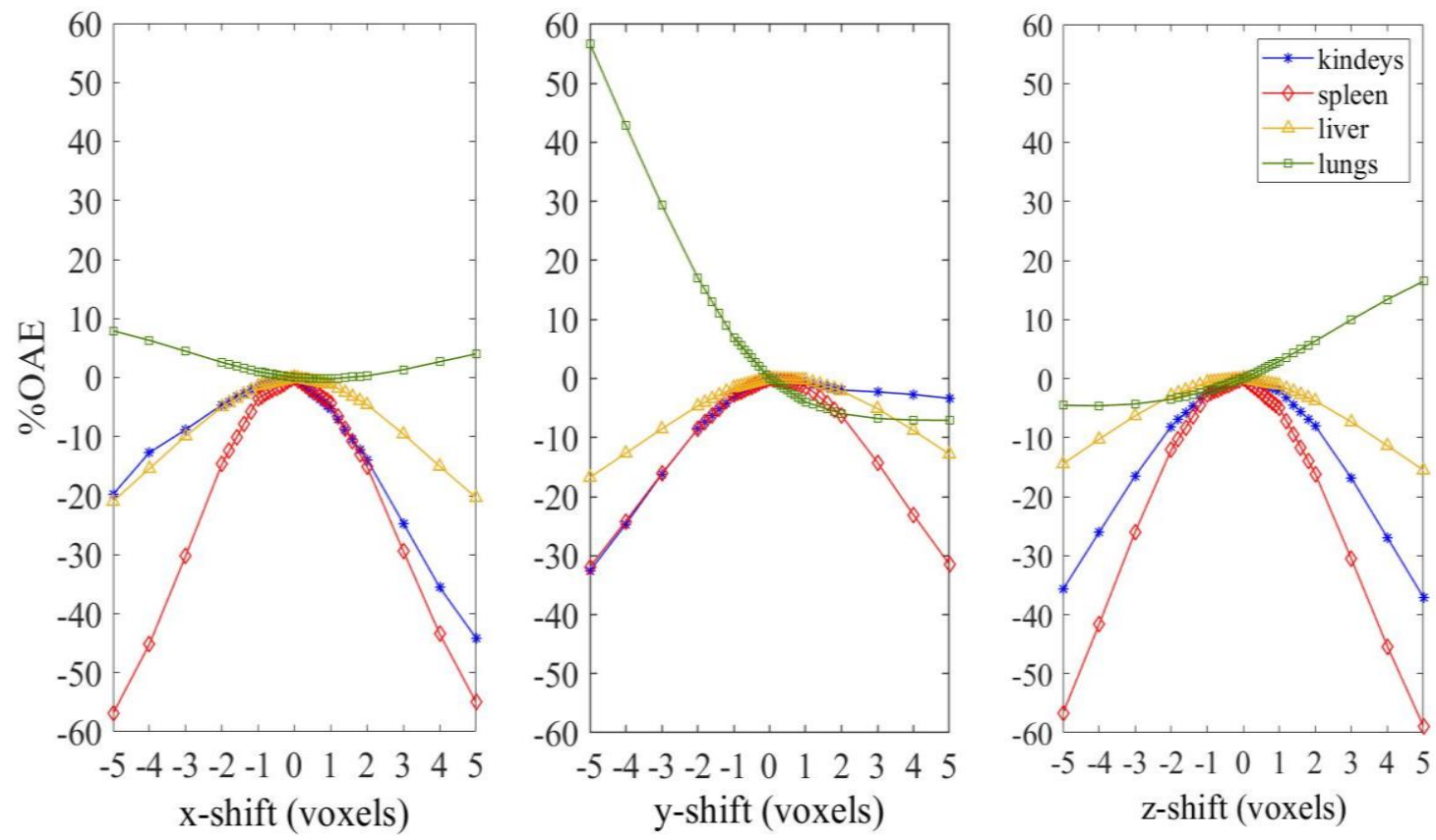

Figure 5: \%OAE introduced by shifting $\mathrm{CT}$ images from -5 to 5 voxels for the mismatched VOI delineation effects. Left: $\mathrm{x}$-direction; middle: $\mathrm{y}$-direction; right: $\mathrm{z}$-direction. 
Table 2: Mean \%OAE and standard deviation of 9 phantoms for mismatched VOI delineation effect.

\begin{tabular}{|l|l|l|l|l|l|}
\hline \multicolumn{2}{|c|}{} & kidneys & spleen & liver & lungs \\
\hline \multirow{2}{*}{ x-shift } & -1 voxel & $-1.08 \% \pm 1.24 \%$ & $-2.22 \% \pm 0.92 \%$ & $-1.47 \% \pm 0.24 \%$ & $0.59 \% \pm 0.31 \%$ \\
\cline { 2 - 6 } & -2 voxels & $-3.17 \% \pm 1.65 \%$ & $-10.02 \% \pm 3.00 \%$ & $-4.86 \% \pm 0.54 \%$ & $1.42 \% \pm 0.93 \%$ \\
\cline { 2 - 6 } & -5 voxels & $-16.37 \% \pm 3.24 \%$ & $-42.83 \% \pm 7.94 \%$ & $-19.79 \% \pm 2.01 \%$ & $4.82 \% \pm 3.07 \%$ \\
\hline \multirow{3}{*}{ y-shift } & -1 voxel & $-4.07 \% \pm 1.16 \%$ & $-1.81 \% \pm 0.94 \%$ & $-1.21 \% \pm 0.37 \%$ & $4.77 \% \pm 1.41 \%$ \\
\cline { 2 - 6 } & -2 voxels & $-9.58 \% \pm 1.86 \%$ & $-6.43 \% \pm 1.80 \%$ & $-3.88 \% \pm 0.83 \%$ & $12.01 \% \pm 3.97 \%$ \\
\cline { 2 - 6 } & -5 voxels & $-30.97 \% \pm 4.14 \%$ & $-26.43 \% \pm 3.57 \%$ & $-14.98 \% \pm 2.07 \%$ & $42.81 \% \pm 6.38 \%$ \\
\hline z-shift & -1 voxel & $-1.94 \% \pm 0.45 \%$ & $-3.65 \% \pm 1.00 \%$ & $-0.84 \% \pm 0.23 \%$ & $-1.86 \% \pm 0.47 \%$ \\
\cline { 2 - 6 } & -2 voxels & $-7.15 \% \pm 1.71 \%$ & $-13.64 \% \pm 2.69 \%$ & $-3.11 \% \pm 0.45 \%$ & $-3.25 \% \pm 1.24 \%$ \\
\cline { 2 - 6 } & -5 voxels & $-32.46 \% \pm 8.03 \%$ & $-55.93 \% \pm 6.58 \%$ & $-13.83 \% \pm 1.06 \%$ & $-6.00 \% \pm 3.94 \%$ \\
\hline
\end{tabular}

\section{3.C. The Mismatched Registration Effect}

Figure 6 shows the \%TIAE results for different numbers of SPECT and CT mismatch existed

273 in a 3-time point study. For sequential imaging sessions, errors increased when the frequency of

274 mismatches between SPECT and CT images increased, e.g., \%TIAE for kidneys was

$2756.47 \% \pm 4.68 \%, 9.60 \% \pm 5.14 \%$, and $14.25 \% \pm 7.07 \%$ respectively for 1-, 2-, and 3-time point

276 SPECT/CT mismatches. Significant differences were observed between mismatch at 1 and 3 time

277 points for all target organs. 


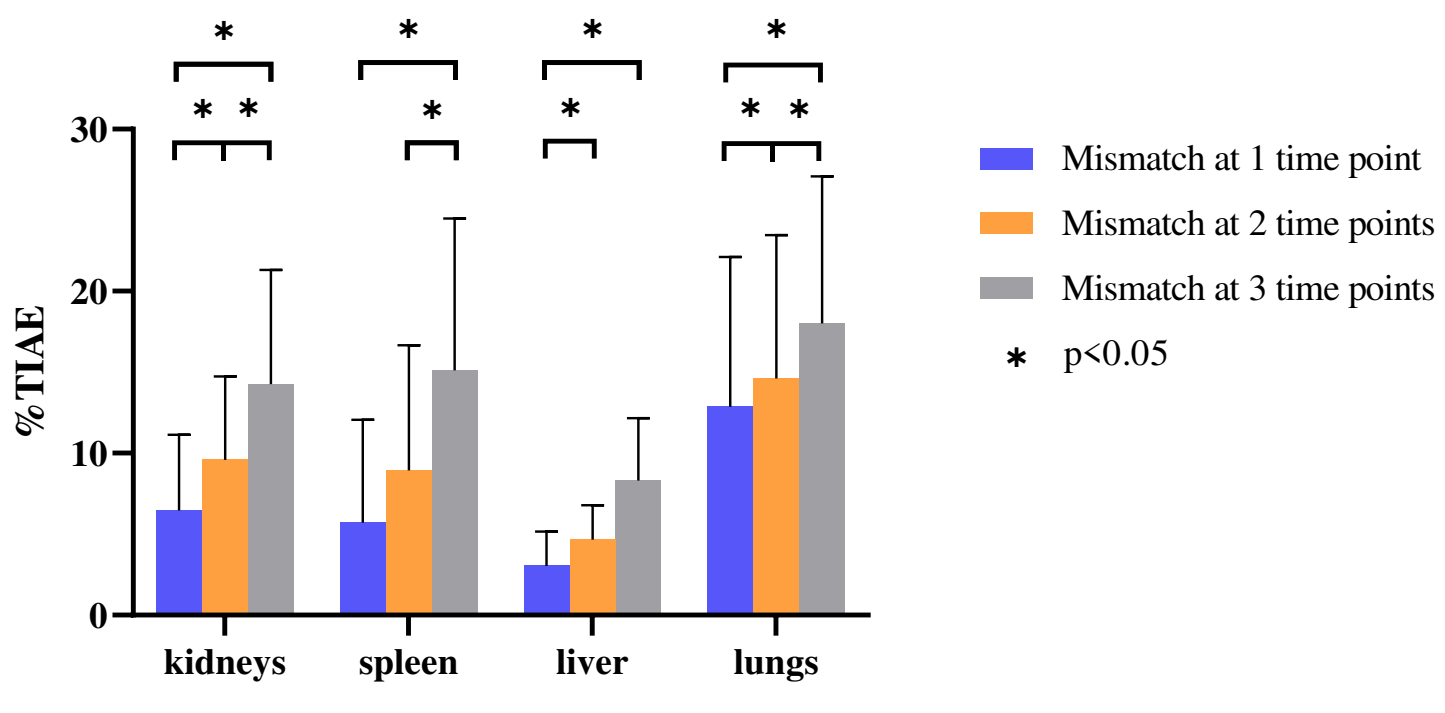

279 Figure 6: \%TIAE for target organs with 1-, 2-, and 3-time-points SPECT/CT mismatch. All

280 results represent the average of 9 phantoms and error bars show the standard deviation.

Figure 7 shows the results of using the different time point CT as "reference image" for registration for sequential CT images to then register the corresponding SPECT images. There was no statistically significant difference among \% TIAE for the CT reference at different time points, no matter when using mismatched or matched $\mathrm{CT}$ as registration reference. The \%TIAE was generally larger for the mismatched CT registration (Figure 7a) as compared to matched CT

287 registration (Figure $7 \mathrm{~b}$ ). The \% TIAE was smaller when mismatch occurs at 1- or 24-hr as compared 288 to 144-hr time point as the registration reference in this study (Figure 7a). 


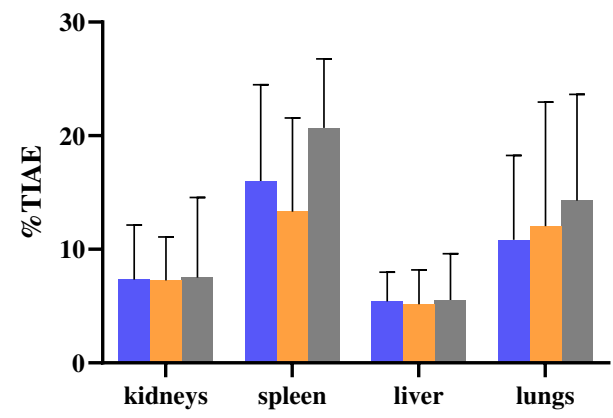

(a)

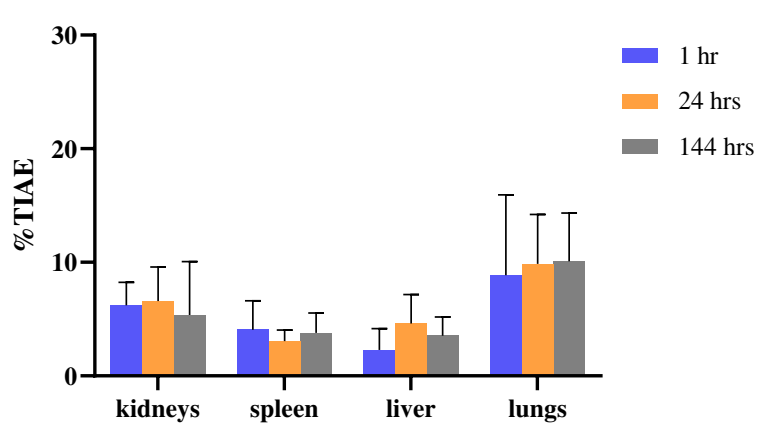

(b)

291 Figure 7: \%TIAE for target organs when using (a) mismatched and (b) matched CT image at 292 different time points as registration reference. All results represent the average of 9 phantoms and 293 error bars show the standard deviation.

Figure 8 compares the \%TIAE results for SPECT and CT mismatch for AC, VOI and registration effects. The \% TIAE for the AC effect was the smallest while the VOI effect for the 3297 time-point mismatch was the largest for all organs, e.g., 1.13\% $\pm 0.90 \%, 37.61 \% \pm 5.08 \%$ and $14.25 \% \pm 7.07 \%$ respectively for mismatched AC, VOI (3-time-point mismatch) and registration 299 effects for kidneys. Even for VOI mismatch occurred in 1-time point, i.e., target organs mapped 300 out 1 time using mismatched VOI from the TIA images, its \%TIAE was still generally larger than 301 3-time point AC and registration mismatch effects for most organs except for the lungs, where 302 registration mismatch affected more on their TIA estimation. In addition, the \%TIAE for the 3303 time-point mismatched VOIs effect was larger as compared to the 1-time-point mismatched VOIs 304 for all organs as expected, e.g., \% TIAE for spleen was $44.86 \% \pm 4.60 \%, 22.94 \% \pm 10.29 \%$ for the 305 3- and 1-time point VOI mismatch respectively. Significant differences were observed among 306 different effects except for the registration and 1-time-point VOI mismatch effect. 


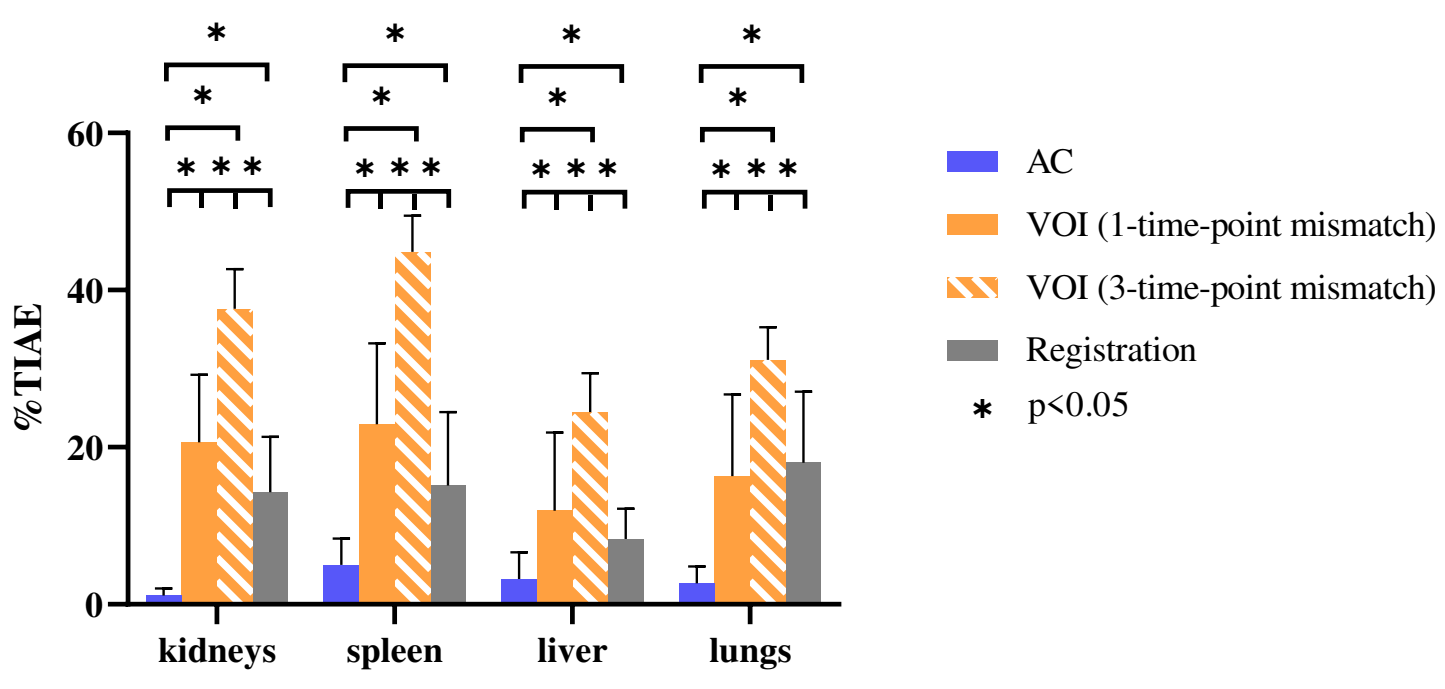

308 Figure 8: \% TIAE for different target organs with the AC, VOI and registration mismatch effects.

309 All results represent the average of 9 phantoms and error bars show the standard deviation.

\section{Discussion}

In this work, the errors of mismatches caused by patient movement between CT and SPECT

313 scans at the same time point were evaluated systematically, including the \%OAE for AC and VOI

314 effects at one scanning time point and the \%TIAE from sequential images taken at different time

315 points. All results showed that the mismatches between CT and SPECT images influence activity

316 estimation and will further affect the TIA estimation. Goetze et al. [14] demonstrated the

317 misregistration frequently occurred between SPECT and CT images in myocardial perfusion

318 SPECT/CT, i.e., about two thirds of the clinical cases showed more than one voxel of

319 misregistration. They demonstrated that the mean misregistration was $10.2 \pm 4.3 \mathrm{~mm}$, with a range

320 of $0-25.1 \mathrm{~mm}$. Thus, besides fixed range of mismatch, CT images in this study were shifted as

321 compared to the SPECT images within the mean and maximal range (2.31 voxels, i.e., $10.2 \mathrm{~mm}$

322 and 5.67 voxels, i.e., $25.1 \mathrm{~mm}$ respectively) according to their study.

323 The \%OAE results revealed the trends and magnitude of errors in activity estimates resulting 324 from AC and VOI effects. The AC errors of the organs near ribs showed a larger impact compared 
325 with the organs far from the ribs. This could be attributed to the larger attenuation coefficient of 326 bone as compared to the soft tissues. For instance, when the CT maps is shifted to the right, the ribs 327 could be moved into the liver region and would cause over attenuation compensation thus activity 328 overestimation in the liver. On the contrary, if the CT is shifted to the left, the background could 329 move into the liver, leading to the underestimation of the liver activity. Moreover, the ribs 330 movement is not much in the y-direction as compared to other directions, thus the \% OAE is 331 relatively smaller for the AC mismatch effect in this direction.

332 For VOI mismatch effect, \%OAE was larger for small organs, such as kidneys and spleen, and 333 low uptake organs that are close to the high uptake organs, e.g., the lungs. For example, for VOI 334 mismatch according to clinical reference, the \%OAE can reach $-11.94 \% \pm 10.34 \%$ for the spleen. 335 All target organs except the lungs showed activity underestimation since the adjacent region had 336 lower activities. Overestimation was expected for the lungs, since they were near to the heart and 337 liver with higher activity. These results were in accordance with the results from another study [13]. For registration mismatch effect, we evaluated the errors for using mismatched CT images at 339 different time points as the "reference image" and different numbers of mismatch occurred in 340 sequential SPECT/CT. No significant difference was observed when using different time point 341 images for registration reference, probably attributed to the fact that CTs at different imaging points 342 possess similar image intensity while it is different in sequential SPECT images for SPECT-based 343 registration [5]. For a 3-time point imaging study, \%TIAE is $>10 \%$ for kidneys, spleen and lungs 344 when SPECT and CT mismatch accumulates for 3 SPECT/CT sessions (Figure 6).

345 The VOI errors were larger than the AC errors in both organ activity and TIA estimation, 346 especially for small organs, such as kidneys and spleen. The \%ODE for kidneys and spleen were $3479.14 \% \pm 8.47 \%$ and $-11.94 \% \pm 10.34 \%$ for 1 -time point SPECT and CT random VOI mismatch, 348 which were further accumulated in TIA calculation by sequential mismatched images, i.e., 349 the \%TIAE for kidneys and spleen were $37.61 \% \pm 5.08 \%$ and $44.86 \% \pm 4.60 \%$ for 3-time-point 350 mismatched VOI and $20.62 \% \pm 8.61 \%$ and $22.94 \% \pm 10.29 \%$ for 1-time-point mismatched VOI 
351 respectively. The VOI errors were generally largest, followed by registration and AC errors in TIA 352 estimation.

353 Simulation provides an effect means for evaluation with known truth, i.e., organ activity and 354 time integrated activity in this study. Moreover, different mismatch schemes can be modeled based 355 on the clinical reference [14]. In this study, rigid mismatch is modelled between the SPECT and 356 CT, which should be a legitimate assumption for voluntary motion happened between the same 357 time point SPECT and CT due to the relatively short time gap. However, nonrigid motion 358 deformation may exist between the same time point SPECT and CT from involuntary motion, e.g., 359 respiratory motion, and also impact the dosimetric accuracy. For example, liver and lungs mis360 registration between the mismatched SPECT/PET and CT due to respiration in Y-90 microsphere 361 treatment planning or dose verification for primary or metastatic cancers in liver is commonly 362 observed in the clinics, posing uncertainties for then dosimetric calculations. Associate results are 363 published in our recent study [26]. However, registration between mismatched SPECT and CT due 364 to respiration could be challenging due to the substantially inherent motion blur thus unclear organ 365 boundaries from the static emission images. Respiratory gating and motion compensation could be 366 a possible solution [27]. Although restraints and careful position could reduce patients' movement, 367 our results indicate that non-negligible mismatches between SPECT and CT images at the same 368 time point could affect the dosimetric accuracy $>40 \%$ especially for small organs and low uptake 369 organs (Figure 8), while the accuracy of organ activity estimation for quantitative SPECT exceeds $37090 \%$ with appropriate compensation [28]. Thus, alleviating the mismatch between transmission and 371 emission images by registration is necessary to improve the AC, VOI delineation and the then CT372 based registration among serial scans for TRT dosimetry. Lesions are not simulated in this study as 373 for the maximal tolerated dose regime, absorbed dose for critical organs are more important. 374 However, SPECT and CT mismatch impact on lesions are expected to be similar or even more 375 severe to small organs, i.e., spleen in this study. Although this study is based on simulations with 
376 In-111 Zevalin distribution, results should be applicable to other common clinical applications such 377 as Lu-177 tracers nowadays.

\section{5. Conclusion}

This work studied the mismatch effects between SPECT and CT scans at the same time point, 381 including the activity estimation errors caused by the AC and VOI delineation, and TIA estimation 382 errors from AC, VOI delineation and registration from sequential SPECT/CT scans. Our results 383 showed that dosimetric estimation errors increase as the mismatch magnitude increases. It was 384 observed that the VOI errors were the largest, followed by registration and AC errors for dosimetric 385 estimations, especially for the small organs and low uptake organs in this study. Quantitative errors 386 could reach $40 \%$ for SPECT and CT mismatch modeled according to a clinical reference.

387 Registration between the same time point SPECT and CT is recommended to enhance dosimetric 388 accuracy.

390 Abbreviations

391 SPECT/CT: Single photon emission computed tomography/computed tomography; 392 SPECT: Single photon emission computed tomography; CT: Computed tomography; AC: 393 Attenuation correction; VOI: Volume-of-interest; GCDR: Geometric collimator-detector394 response; TIA: Time-integrated activity; OAE: Organ activity error; TIAE: Time395 integrated activity error; TRT: Targeted radionuclide therapy; TAC: time-activity curve; 396 XCAT: 4D digital extended cardiac torso; MEGP: Medium energy general purpose; ESSE: 397 Effective source scatter estimation; OS-EM: Ordered subset expectation maximization.

\section{Acknowledgements}


400 Not applicable.

401

402 Authors' contributions

403 Yingqing Lyu and Greta Mok were both the primary writers of the manuscript. Yingqing

404 Lyu was mainly responsible for phantom generation, data collection and analysis while

405 Greta Mok and Yue Chen were responsible for the simulation design, data interpretation

406 and study integration.

407

408 Funding

409 This work was supported by research grants from Science and Technology Development

410 Fund of Macau (0091/2019/A2) and Natural Science Foundation of China (81922080).

411

412 Availability of data and materials

413 The datasets used and analyzed during the current study are available from the 414 corresponding author on reasonable request.

415

416 Ethics approval and consent to participate

417 Not applicable.

418

419 Consent for publication

420 Not applicable.

421

422 Competing interests 
423 The authors declare that they have no competing interests.

\section{Reference:}

427 1. Gudkov SV, Shilyagina NY, Vodeneev VA, Zvyagin AV. Targeted radionuclide therapy of 428 human tumors. Int J Mol Sci. 2016;17:33. https://doi.org/10.3390/ijms17010033.

$4292 . \quad$ Kassis Al, Adelstein SJ. Radiobiologic principles in radionuclide therapy. J Nucl Med. 430 2005; 46 Suppl 1:4S-12S.

431 3. Li T, Ao ECl, Lambert B, Brans B, Vandenberghe S, Mok GSP. Quantitative Imaging for 432 Targeted Radionuclide Therapy Dosimetry - Technical Review. Theranostics. 2017;7:4551-65. 433 https://doi.org/10.7150/thno.19782.

434 4. Li T, Wu NY, Song N, Mok GSP. Evaluation of sequential SPECT and CT for targeted 435 radionuclide therapy dosimetry. Ann Nucl Med. 2018;32:34-43.

436 5. Li T, Mok GSP. Technical Note: Virtual CT for reducing CT dose in targeted radionuclide 437 therapy dosimetry. Med Phys. 2018;45:5138-44.

$438 \quad 6$ Schillaci O, Spanu A, Tagliabue L, Filippi L, Danieli R, Palumbo B, et al. SPECT/CT with a 439 hybrid imaging system in the study of lower gastrointestinal bleeding with technetium-99m red 440 blood cells. Q J Nucl Med Mol Imaging. 2009;53:281-9.

441 7. Zhang D, Yang B-H, Wu NY, Mok GSP. Respiratory average CT for attenuation correction 442 in myocardial perfusion SPECT/CT. Ann Nucl Med. 2017;31:172-80.

443 8. Nakamoto Y, Tatsumi M, Cohade C, Osman M, Marshall LT, Wahl RL. Accuracy of image 444 fusion of normal upper abdominal organs visualized with PET/CT. Eur J Nucl Med Mol Imaging. 445 2003;30:597-602.

446 9. Sun T, Mok GS. Techniques for respiration-induced artifacts reductions in thoracic

447 PET/CT. Quant Imaging Med Surg. 2012;2:46-52. https://doi.org/10.3978/j.issn.2223-

$448 \quad 4292.2012 .02 .01$.

449 10. Ho CY, Wu T-H, Mok GSJNmc. Interpolated average CT for PET attenuation correction in 450 different lesion characteristics. Nucl Med Commun. 2016;37:297-306.

451 11. Mok GS, Ho CY, Yang B-H, Wu T-HJJoNC. Interpolated average CT for cardiac PET/CT 452 attenuation correction. J Nucl Cardiol. 2016;23:1072-9.

453 12. Sun T, Wu TH, Wang SJ, Yang BH, Wu NY, Mok GS. Low dose interpolated average CT for 454 thoracic PET/CT attenuation correction using an active breathing controller. Med Phys. $455 \quad 2013 ; 40: 102507$.

456 13. He B, Frey EC. The impact of 3D volume of interest definition on accuracy and precision 457 of activity estimation in quantitative SPECT and planar processing methods. Phys Med Biol.

458 2010;55:3535-44. https://doi.org/10.1088/0031-9155/55/12/017.

459 14. Goetze S, Brown TL, Lavely WC, Zhang Z, Bengel FM. Attenuation correction in 460 myocardial perfusion SPECT/CT: effects of misregistration and value of reregistration. J Nucl 461 Med. 2007;48:1090-5. https://doi.org/10.2967/jnumed.107.040535.

462 15. Sjögreen-Gleisner K, Rueckert D, Ljungberg M. Registration of serial SPECT/CT images for 463 three-dimensional dosimetry in radionuclide therapy. Phys Med Biol. 2009;54:6181-200.

464 16. Segars WP, Sturgeon G, Mendonca S, Grimes J, Tsui BM. 4D XCAT phantom for 465 multimodality imaging research. Med Phys. 2010;37:4902-15.

466 https://doi.org/10.1118/1.3480985. 
17. He B, Du Y, Segars WP, Wahl RL, Sgouros G, Jacene H, et al. Evaluation of quantitative imaging methods for organ activity and residence time estimation using a population of phantoms having realistic variations in anatomy and uptake. Med Phys. 2009;36:612-9.

470 https://doi.org/10.1118/1.3063156.

471 18. Frey E, Tsui B. A practical projector-backprojector modeling attenuation, detector 472 response, and scatter for accurate scatter compensation in SPECT. Conference Record of the 4731991 IEEE Nuclear Science Symposium and Medical Imaging Conference. 1991;3:1777-81.

474 19. Seo Y, Wong KH, Hasegawa BH. Calculation and validation of the use of effective 475 attenuation coefficient for attenuation correction in In - 111 SPECT. Med Phys. 2005;32:3628-

47635.

477 20. Frey EC, Tsui B. A new method for modeling the spatially-variant, object-dependent 478 scatter response function in SPECT. 1996 IEEE Nuclear Science Symposium Conference Record. 479 1996;2:1082-6.

480 21. Metz CE, Atkins F, Beck RN. The geometric transfer function component for scintillation 481 camera collimators with straight parallel holes. Phys Med Biol. 1980;25:1059-70.

482 22. Yushkevich PA, Piven J, Hazlett HC, Smith RG, Ho S, Gee JC, et al. User-guided 3D active 483 contour segmentation of anatomical structures: significantly improved efficiency and reliability. 484 Neuroimage. 2006;31:1116-28. https://doi.org/10.1016/j.neuroimage.2006.01.015.

485 23. Song N, He B, Frey E. The effect of volume-of-interest misregistration on quantitative 486 planar activity and dose estimation. Phys Med Biol. 2010;55:5483-97.

487 https://doi.org/10.1088/0031-9155/55/18/014.

488 24. Ao EC, Wu NY, Wang SJ, Song N, Mok GS. Improved dosimetry for targeted radionuclide 489 therapy using nonrigid registration on sequential SPECT images. Med Phys. 2015;42:1060-70.

$490 \quad 25 . \quad K l e i n$ S, Staring M, Murphy K, Viergever MA, Pluim JP. Elastix: a toolbox for intensity491 based medical image registration. IEEE Trans Med Imaging. 2009;29:196-205.

492 26. Lu Z, Chen G, Lin KH, Wu TH, Mok GS. Evaluation of Different CT Maps for Attenuation 493 Correction and Segmentation in Static 99mTc - MAA SPECT/CT for 90Y Radioembolization

494 Treatment Planning - a Simulation Study. Med Phys. 2021.

495 27. Bastiaannet R, Viergever MA, de Jong HW. Impact of respiratory motion and acquisition 496 settings on SPECT liver dosimetry for radioembolization. Med Phys. 2017;44:5270-9.

497 28. He B, Du Y, Song X, Segars WP, Frey EC. A Monte Carlo and physical phantom evaluation 498 of quantitative In-111 SPECT. Phys Med Biol. 2005;50:4169-85. 